\title{
Primary deficiency of microsomal triglyceride transfer protein in human abetalipoproteinemia is associated with loss of CD1 function
}

\author{
Sebastian Zeissig, ${ }^{1}$ Stephanie K. Dougan, ${ }^{1}$ Duarte C. Barral, ${ }^{2}$ Yvonne Junker, ${ }^{3}$ Zhangguo Chen, ${ }^{1}$ \\ Arthur Kaser, ${ }^{4}$ Madelyn Ho, ${ }^{1}$ Hannah Mandel, ${ }^{1}$ Adam McIntyre, ${ }^{5}$ Susan M. Kennedy, ${ }^{6}$ \\ Gavin F. Painter, ${ }^{7}$ Natacha Veerapen, ${ }^{8}$ Gurdyal S. Besra, ${ }^{8}$ Vincenzo Cerundolo, ${ }^{9}$ Simon Yue, ${ }^{10}$ \\ Sarah Beladi, ${ }^{2}$ Samuel M. Behar, ${ }^{2}$ Xiuxu Chen, ${ }^{11}$ Jenny E. Gumperz,, ${ }^{11}$ Karine Breckpot, ${ }^{12}$ \\ Anna Raper, ${ }^{13}$ Amanda Baer, ${ }^{13}$ Mark A. Exley, ${ }^{10}$ Robert A. Hegele, ${ }^{5}$ Marina Cuchel, ${ }^{13}$ \\ Daniel J. Rader, ${ }^{13}$ Nicholas O. Davidson, ${ }^{6}$ and Richard S. Blumberg ${ }^{1}$
}

${ }^{1}$ Division of Gastroenterology, Hepatology, and Endoscopy and 2Division of Rheumatology, Immunology, and Allergy, Brigham and Women's Hospital, Harvard Medical School, Boston, Massachusetts, USA. ${ }^{3}$ Division of Gastroenterology and Hepatology, Beth Israel Deaconess Medical Center, Harvard Medical School, Boston, Massachusetts, USA. ${ }^{4}$ Department of Medicine II, Medical University Innsbruck, Innsbruck, Austria. ${ }^{5}$ Robarts Research Institute and Schulich School of Medicine and Dentistry, University of Western Ontario, London, Ontario, Canada.

${ }^{6}$ Gastroenterology Division, Washington University School of Medicine, St. Louis, Missouri, USA. ${ }^{7}$ Carbohydrate Chemistry Team, Industrial Research Limited, Lower Hutt, New Zealand. ${ }^{8}$ School of Biosciences, University of Birmingham, Birmingham, United Kingdom. 9MRC Human Immunology Unit,

Weatherall Institute of Molecular Medicine, John Radcliffe Hospital, Oxford, United Kingdom. ${ }^{10} \mathrm{Cancer} \mathrm{Biology} \mathrm{Program,} \mathrm{Division} \mathrm{of} \mathrm{Hematology} \mathrm{and} \mathrm{Oncology,}$ Beth Israel Deaconess Medical Center, Harvard Medical School, Boston, Massachusetts, USA. ${ }^{11}$ Department of Medical Microbiology and Immunology, University of Wisconsin School of Medicine and Public Health, Madison, Wisconsin, USA. ${ }^{12}$ Laboratory of Molecular and Cellular Therapy, Department of Physiology-Immunology, Medical School of the Vrije Universiteit Brussel, Brussels, Belgium. ${ }^{13}$ Cardiovascular Medicine Division, University of Pennsylvania School of Medicine, Philadelphia, Pennsylvania, USA.

\begin{abstract}
Abetalipoproteinemia $(\mathrm{ABL})$ is a rare Mendelian disorder of lipid metabolism due to genetic deficiency in microsomal triglyceride transfer protein (MTP). It is associated with defects in MTP-mediated lipid transfer onto apolipoprotein B (APOB) and impaired secretion of APOB-containing lipoproteins. Recently, MTP was shown to regulate the CD1 family of lipid antigen-presenting molecules, but little is known about immune function in ABL patients. Here, we have shown that $A B L$ is characterized by immune defects affecting presentation of self and microbial lipid antigens by group 1 (CD1a, CD1b, CD1c) and group 2 (CD1d) CD1 molecules. In dendritic cells isolated from ABL patients, MTP deficiency was associated with increased proteasomal degradation of group 1 CD1 molecules. Although CD1d escaped degradation, it was unable to load antigens and exhibited functional defects similar to those affecting the group 1 CD1 molecules. The reduction in CD1 function resulted in impaired activation of CD1-restricted $T$ and invariant natural killer $T$ (iNKT) cells and reduced numbers and phenotypic alterations of iNKT cells consistent with central and peripheral CD1 defects in vivo. These data highlight MTP as a unique regulator of human metabolic and immune pathways and reveal that $A B L$ is not only a disorder of lipid metabolism but also an immune disease involving CD1.
\end{abstract}

\section{Introduction}

Abetalipoproteinemia $(\mathrm{ABL})$ is a rare autosomal recessive disorder caused by mutations in the gene encoding microsomal triglyceride transfer protein (MTP) (1). MTP is an ER-resident protein that transfers triglycerides, cholesteryl esters, and phospholipids onto apolipoprotein $\mathrm{B}$ (APOB), thereby promoting the secretion of chylomicrons and very low density lipoproteins (VLDLs) from enterocytes and hepatocytes, respectively $(1,2)$. Consequently, patients with ABL exhibit intestinal lipid malabsorption, low plasma lipid levels, hepatic steatosis, and neurological and ophthalmological symptoms (1).

Authorship note: Nicholas O. Davidson and Richard S. Blumberg are co-senior authors.

Conflict of interest: M.A. Exley owns equity in NKT Therapeutics Inc. and has a consulting arrangement with NKT Therapeutics Inc. D.J. Rader owns a small amount of equity in Aegerion Pharmaceuticals, which is developing MTP inhibitors for LDL reduction.

Citation for this article: JClin Invest. 2010;120(8):2889-2899. doi:10.1172/JCI42703.
Similar observations have been made in MTP-deficient mice, confirming the crucial role of MTP in the pathogenesis of $\operatorname{ABL}(3,4)$.

Recent studies have shown that MTP also regulates CD1, a family of lipid antigen-presenting molecules consisting of group 1 (CD1a, CD1b, CD1c) and 2 (CD1d) CD1 molecules in humans (5). CD1 is synthesized in the ER, where it is loaded with endogenous (self) lipid antigens that are presented to CD1-restricted T cells after reaching the plasma membrane (5). Subsequently, CD1 traffics through the endolysosomal system, where endogenous antigens are exchanged for exogenous lipid antigens, which are presented to $\mathrm{T}$ cells after recycling to the cell surface (5). It has recently been shown that MTP can directly transfer phospholipids onto CD1d, suggesting a role for MTP in transfer of endogenous lipids onto CD1 during its biosynthesis in the ER (6). Furthermore, deletion of MTP in parenchymal cells of mice as well as chemical inhibition of MTP in human cells leads to strongly impaired CD1-restricted antigen presentation, demonstrating a critical role of MTP in CD1 function (6-9). 
A
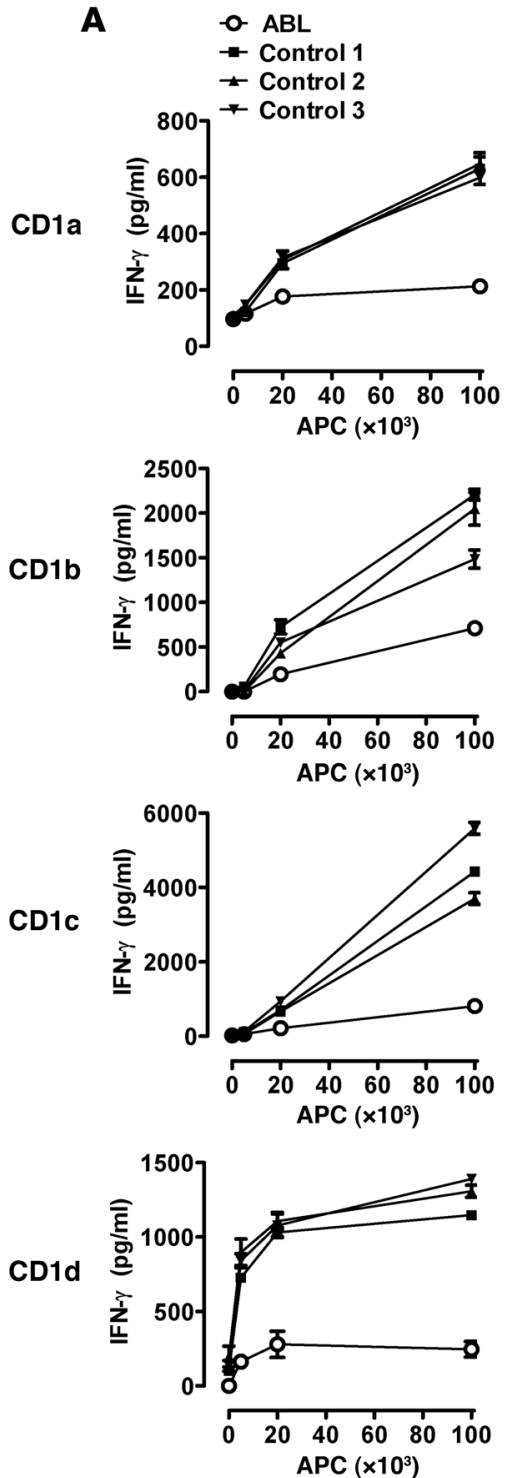

B
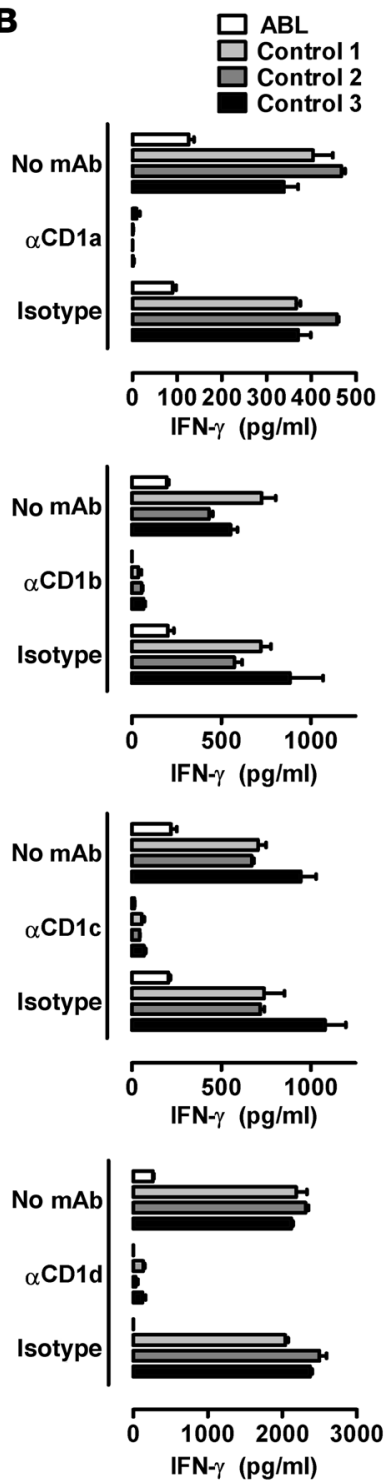
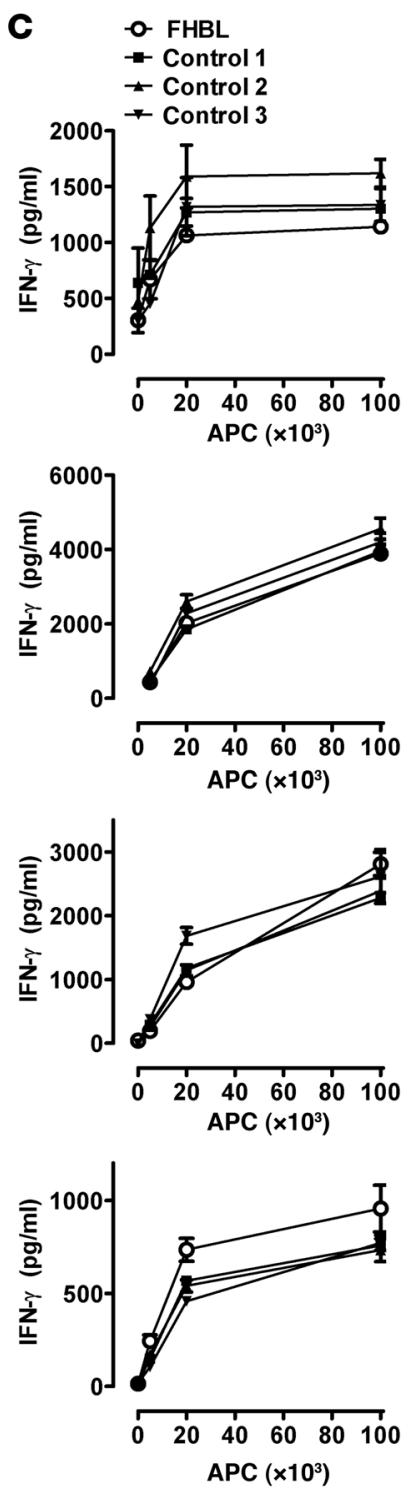

Figure 1

Impaired presentation of self-derived antigens in ABL but not FHBL. Monocyte-derived DCs from healthy controls and $\mathrm{ABL}$ patients (A and $\mathbf{B})$ or FHBL patients $(\mathbf{C})$ were cultured at the indicated concentrations with $5 \times 10^{4}$ CD1a-, CD1b-, and CD1c-restricted T cells or CD1d-restricted iNKT cells. Cytokine release of T cells was determined after 18-20 hours of coculture by ELISA. Culture of DCs or T cells alone did not lead to detectable cytokine production. CD1 restriction is demonstrated by blocking of the T cell response by anti-CD1 antibodies (B). One representative experiment is shown. Two additional experiments with cells from independent ABL, FHBL and control subjects gave comparable results.

CD1d is the best-characterized CD1 family member and is known for its activation of a unique subset of $\mathrm{T}$ cells called natural killer T (NKT) cells. Upon activation by CD1-bearing stimulatory self or foreign lipid antigens, NKT cells secrete a broad array of cytokines, which leads to downstream activation of the innate and adaptive immune system (10). Studies in mice have suggested a central role for NKT cells in antimicrobial, anticancer, and autoinflammatory immune responses $(10,11)$. While less is known about group 1 CD1-restricted T cells due to their absence in mice, most antigens that are known to activate these $\mathrm{T}$ cells are derived from mycobacteria, particularly Mycobacterium tuberculosis (12).

In the current study, we have investigated CD1 function in patients with $\mathrm{ABL}$ and found that primary genetic deficiency of
MTP in ABL is associated with loss of CD1 function. We thus describe the first human disease to our knowledge characterized by defects in all antigen-presenting CD1 family members.

\section{Results}

Defect in CD1-restricted presentation of self-derived antigens in $A B L$. Antigen presentation was analyzed by coculture of CD1-restricted $\mathrm{T}$ cell clones with APCs obtained from peripheral blood of ABL patients $(n=6$, Supplemental Table 1 ; supplemental material available online with this article; doi:10.1172/JCI42703DS1) or healthy controls $(n=33)$. To determine whether changes in CD1 presentation are specifically caused by MTP deficiency, we analyzed 6 patients with familial hypobetalipoproteinemia (FHBL) caused 
A

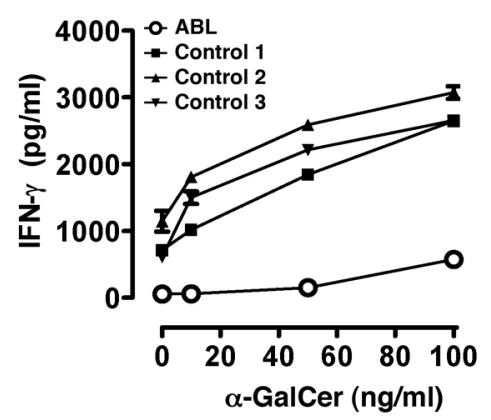

D

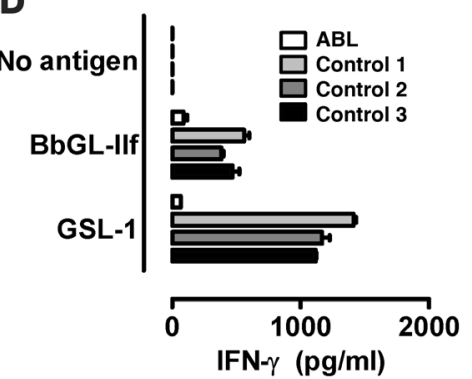

G

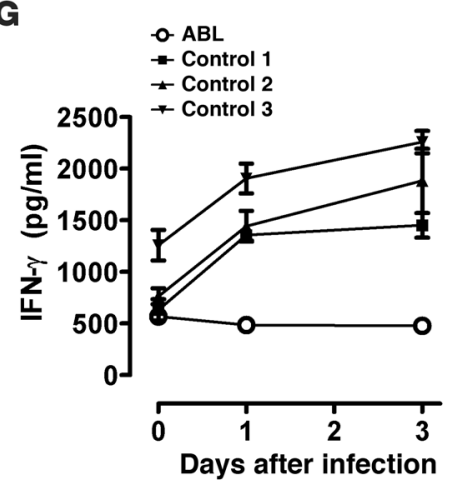

B $\bigcirc A B L$

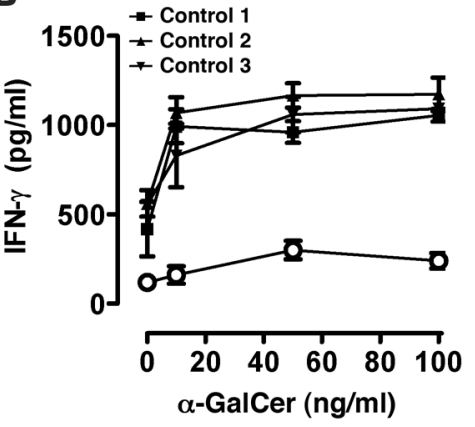

E

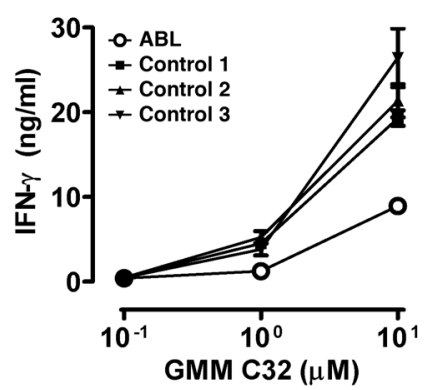

H

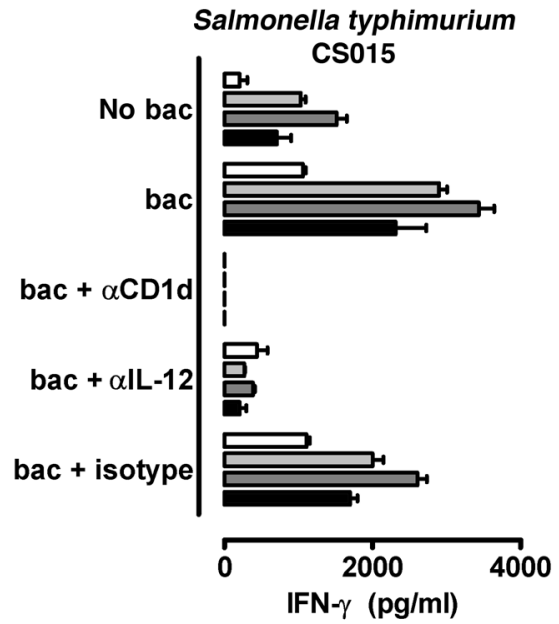

C

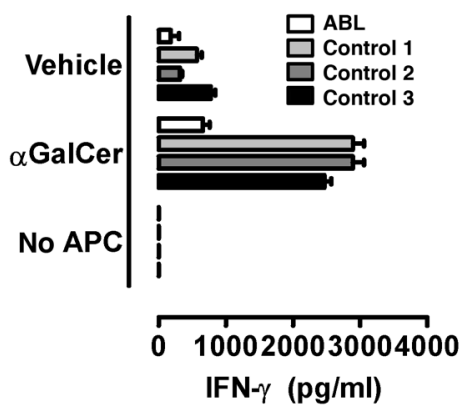

F

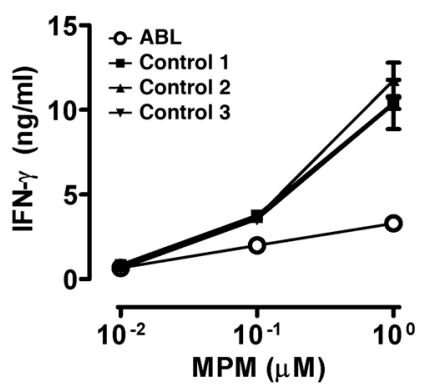

Staphylococcus aureus

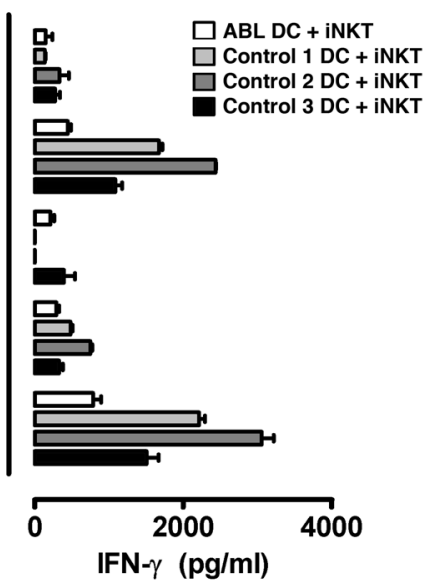

Figure 2

Impaired presentation of exogenous antigens in ABL. DCs (A and $\mathbf{D}-\mathbf{H})$, monocytes $(\mathbf{B})$ or B cells $(\mathbf{C})\left(2 \times 10^{4}\right)$ were cocultured with $1 \times 10^{5}$ CD1drestricted NKT cells $(\mathbf{A}-\mathbf{D}$ and $\mathbf{H})$ or CD1b- $(\mathbf{E})$ or CD1c-restricted T cells $(\mathbf{F})$ in the presence of $\alpha$ GalCer $(\mathbf{A}-\mathbf{C}, 100 \mathrm{ng} / \mathrm{ml}$ in $\mathbf{C})$, GMM $(\mathbf{E})$, mannosyl-phosphomycoketide (MPM; F), BbGL-Ilf (10 $\mathrm{g} / \mathrm{ml}, \mathbf{D})$, or GSL-1 (10 $\mu \mathrm{g} / \mathrm{ml}, \mathbf{D})$. In G, DCs were cocultured with CD1c-restricted T cells at the indicated days after infection with M. tuberculosis H37Rv. In H, DCs were cocultured with CD1d-restricted iNKT cells in the presence of the indicated heat-killed bacteria (MOI of 20). In some cases, blocking antibodies against CD1d or IL-12 were added. Cytokine release of T cells was determined after 18-20 hours of coculture by ELISA. Culture of DCs or T cells alone did not lead to detectable cytokine production. One representative experiment is shown. Two additional experiments with cells from independent ABL and control subjects showed comparable results. bac, bacteria.

by heterozygous $(n=5)$ or compound heterozygous mutations $(n=1)$ in $A P O B$ (Supplemental Table 1). In addition, we analyzed one severely hypolipidemic FHBL subject with a combination of heterozygous mutations in $M T T P, S A R 1 B$, and $A P O B$. Since APOB, like MTP, is a requisite component for intestinal and hepatic lipid transport but does not regulate CD1 function, FHBL patients display similar serum lipid changes similar to those observed in $\mathrm{ABL}$ but are not expected to show alterations in CD1 function.

CD1 molecules present self antigens, and, at least in the case of human CD1d, the majority of these antigens seem to be acquired in the secretory pathway (ER, Golgi apparatus) (13). Since MTP resides in the ER and can directly transfer phospholipids onto CD1 (6), we hypothesized that it might be critical for presentation of self antigens by CD1. Indeed, ABL patients exhibited a severe defect in self antigen presentation by all human CD1 isoforms (Figure 1, A and B, and Supplemental Figure 1A). In contrast, FHBL patients, including a patient with compound heterozygous $A P O B$ mutations and a severely hypolipidemic subject with heterozygous mutations in MTTP, SAR1B, and $A P O B$ exhibited normal CD1-restricted antigen presentation (Figure 1C, Supplemental Figure 1A, 

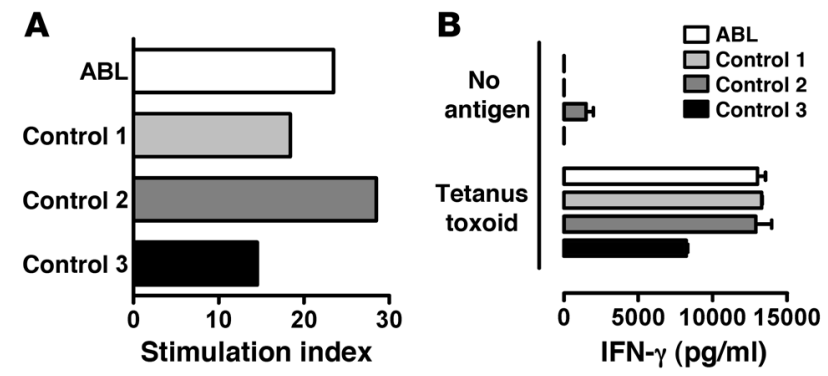

Figure 3

Unaltered $\mathrm{MHC}$ class I- and class II-restricted antigen presentation in $\mathrm{ABL}$ patients. (A) PBMCs $\left(2 \times 10^{5}\right)$ were cultured in the presence or absence of $2 \mu \mathrm{g} / \mathrm{ml}$ CEF peptide mixture on IFN- $\gamma$ ELISPOT plates. Plates were developed after 20 hours of culture, and the stimulation index (spots in the presence of CEF/spots in the absence of CEF) was calculated. (B) DCs $\left(2 \times 10^{4}\right)$ were cocultured with $1 \times 10^{5}$ tetanus toxinreactive HLA-DR-restricted SPF3 T cells in the presence or absence of $25 \mu \mathrm{g} / \mathrm{ml}$ tetanus toxin. Cytokine release of T cells was determined after 18-20 hours of coculture by ELISA. Culture of DCs or T cells alone did not lead to detectable cytokine production. One representative experiment is shown. Two additional experiments with cells from independent $\mathrm{ABL}$ and control subjects showed comparable results.

and Supplemental Figure 2, A-C), confirming that CD1 dysfunction is specifically caused by homozygous MTTP mutations and not by downstream effects on the metabolism of APOB-containing lipoprotein particles. Assessment of CD1d-restricted antigen presentation using cells from the same ABL patient at two different time points confirmed a persistent CD1 defect in ABL (Supplemental Figure 3). These studies together with others (see Supplemental Results) reveal that ABL is associated with a specific defect in CD1 function.

Defect in the presentation of exogenous CD1-restricted antigens in $A B L$. To study whether the CD1 defect extends to the presentation of exogenous antigens, we analyzed presentation of the CD1d model glycolipid antigen $\alpha$-galactosylceramide ( $\alpha$ GalCer) to invariant NKT (iNKT) cells. We found that $\alpha$ GalCer presentation was severely impaired in monocyte-derived DCs, monocytes, and B cells from ABL but not FHBL patients (Figure 2, A-C, Supplemental Figure $1 \mathrm{~B}$, and Supplemental Figure 4, A and B), demonstrating that the CD1d defect in ABL extends to exogenous antigens and is not restricted to certain subtypes of APCs. iNKT cells showed impaired production of both Th1 (IFN- $\gamma$ ) and Th2 (IL-4) cytokines upon stimulation with ABL APCs, confirming that decreased IFN- $\gamma$ levels reflect impaired $\mathrm{T}$ cell activation and not altered $\mathrm{T}$ cell polarization (Supplemental Figure 5).

CD1d-restricted antigen presentation in ABL was broadly disabled in that it affected presentation of antigens preferably ( $\alpha$ GalCer) or exclusively (Gal[ $\alpha 1 \rightarrow 2]$ GalCer) (14) loaded in the endolysosomal compartment, as well as presentation of antigens loaded at the cell surface ( $\alpha$ GalCer C20:2; ref. 15 and Supplemental Figure 6, A and B). In addition, ABL subjects exhibited impaired presentation of $\alpha$ GalCer after cell surface loading of fixed monocytes and showed decreased loading of CD1d with $\alpha$ GalCer, as directly detected by Fab fragments specific for the $\alpha \mathrm{GalCer} /$ CD1d complex (16) (Supplemental Figure 6B and Supplemental Figure 7). Furthermore, we tested relative effects of surface and endosomal compartments in the group 1 CD1 system by investigating CD1b-restricted presentation of glucose monomycolates
(GMMs) with short (C32) or long (C80) tails that load at the cell surface (C32) or require lysosomal acidification for loading (C80) (17). We found that ABL DCs had a stronger defect in the presentation of GMM C32 compared with GMM C80, implicating a defect in proximal compartments and suggesting that the acidic $\mathrm{pH}$ in lysosomes and/or the action of lysosomal lipid editing molecules might provide a mechanism for rescue of CD1b-restricted antigen presentation in the absence of MTP (Supplemental Figure 6C).

CD1 molecules present microbial lipids and contribute to clearance of infections through activation of lipid-reactive T and NKT cells (18). To investigate whether ABL patients show a defect in CD1-mediated presentation of microbial antigens, we examined presentation of antigens derived from Borrelia burgdorferi (BbGL-IIf, ref. 19, CD1d-restricted, Figure 2D), Sphingomonas spp. (GSL-1, ref. 20, CD1d-restricted, Figure 2D), and M. tuberculosis (GMM, ref. 17, CD1b-restricted, Figure 2E; mannosyl-phosphomycoketide [MPM], ref. 21, CD1c-restricted, Figure 2F). All antigens led to significant activation of CD1-restricted $\mathrm{T}$ and NKT clones upon presentation by DCs from healthy controls (Figure 2, D-F) and FHBL patients (Supplemental Figure 1B and Supplemental Figure 4, C-E), including a patient with compound heterozygous $A P O B$ mutations and a severely hypolipidemic subject with heterozygous mutations in MTTP, SAR1B, and APOB (Supplemental Figure 2, $\mathrm{D}-\mathrm{F})$. In contrast, DCs from $\mathrm{ABL}$ patients failed to present CD1restricted microbial antigens to $\mathrm{T}$ and NKT cells (Figure 2, D-F, and Supplemental Figure 1B). Blocking antibodies confirmed CD1 restriction of the respective antigens (data not shown). To investigate whether a similar defect can be observed upon encounter with intact microbial organisms, we studied activation of $T$ cells recognizing $M$. tuberculosis-derived lipids in a CD1c-restricted manner after infection of DCs with live M. tuberculosis (Figure 2G). As observed with purified CD1-dependent lipids, infection of DCs from healthy controls led to significant $\mathrm{T}$ cell activation, while ABL DCs failed to activate T cells (Figure $2 \mathrm{G}$ ).

iNKT cells can be activated not only by direct presentation of microbial lipids but also indirectly by stimulation of pattern recognition receptors on DCs, which activate iNKT cells by DC-derived IL-12 in the presence of CD1d-restricted self antigen presentation $(22,23)$. Given the defect in CD1d-restricted presentation of self antigens, we hypothesized that ABL patients might also show altered indirect iNKT cell activation. Indeed, Salmonella typhimurium and Staphylococcus aureus, neither of which contain known CD1-associating iNKT cell-activating lipids, significantly enhanced iNKT cell activation by DCs from healthy controls but not ABL subjects in a pathway dependent upon IL-12 and CD1d (Figure 2H).

The defect in antigen presentation in $A B L$ is specific for $C D 1$. Several control experiments were performed to exclude nonspecific defects in antigen presentation in ABL. Most importantly, we found that MHC class I- and class II-restricted peptide antigen presentation was normal in ABL subjects (Figure 3), confirming a selective CD1 defect and absence of significant alterations in endolysosomal trafficking and antigen processing. Furthermore, expression of the DC maturation marker CD83 and the costimulatory molecules CD40, CD80, and CD86 did not differ between ABL and controls (Figure 4A and data not shown). In addition, TNF- $\alpha$ and IL-8 secretion by ABL monocytes and DCs upon stimulation with LPS or poly(I:C) was indistinguishable in ABL subjects and controls, confirming the absence of general functional alterations in $\mathrm{ABL}$ APCs (Supplemental Figure 8 and data not shown). Finally, comprehensive analysis of the frequency, phenotype, and function of 

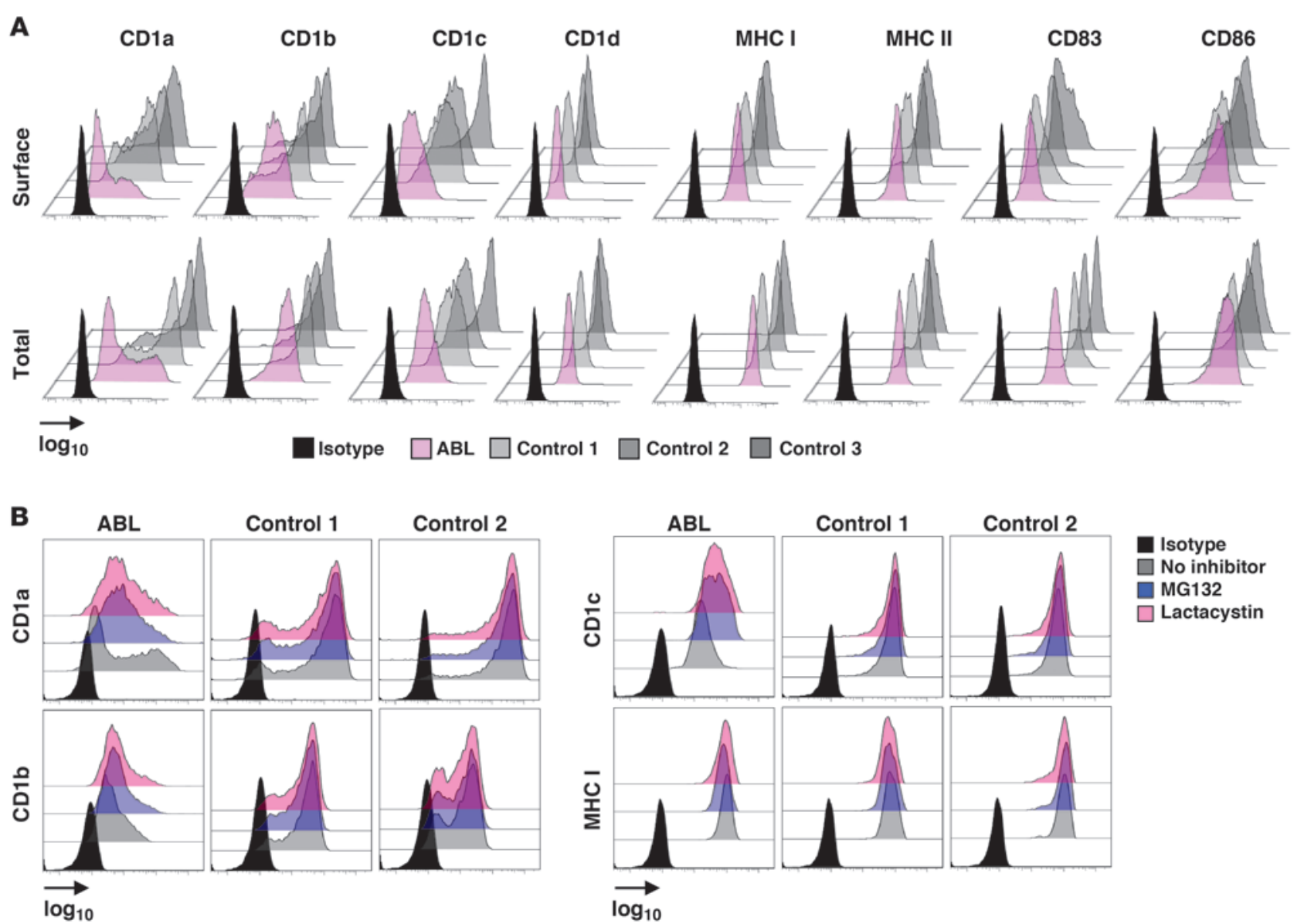

Figure 4

Proteasomal degradation leads to impaired group 1 CD1 expression in ABL. (A) Flow cytometric analysis of expression of the indicated molecules on DCs. For analysis of total (surface and intracellular) expression, cells were permeabilized before antibody staining. For surface staining, cells were stained and then fixed. Shown is the mean fluorescence intensity on a $\log _{10}$ Scale. (B) Flow cytometric analysis of CD1 and $\mathrm{MHC}$ class I expression after culture in the presence or absence of the indicated proteasomal inhibitors (10 $\mu \mathrm{M})$ for 10 hours. Cells were permeabilized before antibody staining to allow for detection of total protein expression. Shown is the mean fluorescence intensity on a log 10 scale. One representative experiment is shown. One (B) and two (A) additional experiments with cells from independent $A B L$ and control subjects showed comparable results.

ABL PBMCs revealed no alterations beyond those related to CD1 dysfunction (Supplemental Results).

MTP deficiency in ABL leads to degradation of group 1 CD1 molecules. To investigate the underlying mechanisms responsible for the CD1 defect in ABL, we determined cell surface expression of CD1 molecules by flow cytometry. DCs from ABL patients exhibited a dramatic decrease in cell surface expression of CD1a and CD1c and, to a lesser degree, of CD1b (Figure 4A). MHC class I and II expression was not changed in ABL (Figure 4A), confirming a selective CD1 defect. Interestingly, CD1d expression on monocytes, DCs, and B cells was unchanged as determined by 3 different monoclonal antibodies (51.1, 42.1, and Nor 3.2; Figure 4 and data not shown), despite a pronounced functional defect and inability to load exogenous antigens (Figure 1, Figure 2, A-D, and Supplemental Figures 6 and 7).

Impaired cell surface expression of CD1 could be due to decreased protein expression or increased internalization of surface protein. To delineate these possibilities, we determined total protein expression using flow cytometry of permeabilized DCs.
ABL DCs showed decreased total CD1a, CD1b, and CD1c levels that correlated with reduction in cell surface expression (Figure 4A). In addition, confocal microscopy showed reduced expression of group 1 CD1 but, where detectable, no alterations in subcellular distribution (Supplemental Figure 9). In accordance with unaltered CD1 function in FHBL, CD1a-d surface and total expression were unchanged (data not shown).

To investigate whether decreased group 1 CD1 expression is due to altered transcription, we analyzed CD1 mRNA expression by quantitative PCR. We found unaltered transcription of CD1a, CD1b, CD1c, $\beta_{2}$-microglobulin, and the nonclassical MHC class I molecule HFE in ABL DCs (Supplemental Figure 10). We therefore investigated the possibility that MTP deficiency leads to proteasomal degradation not only of $\operatorname{APOB}(24,25)$ but also of group 1 CD1 molecules. To this end, we treated DCs with 3 different proteasomal inhibitors, MG132, ALLN, and lactacystin, and determined total CD1 expression by flow cytometry of permeabilized DCs. None of the inhibitors altered CD1 expression in healthy controls, confirming absence of proteasomal CD1 degradation 
A

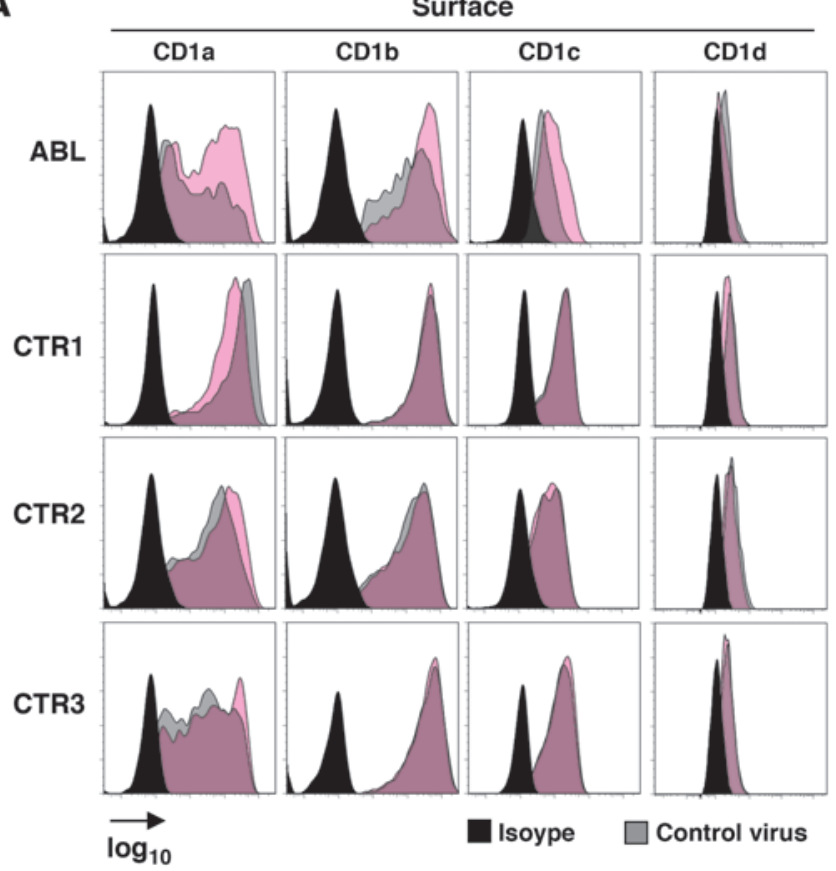

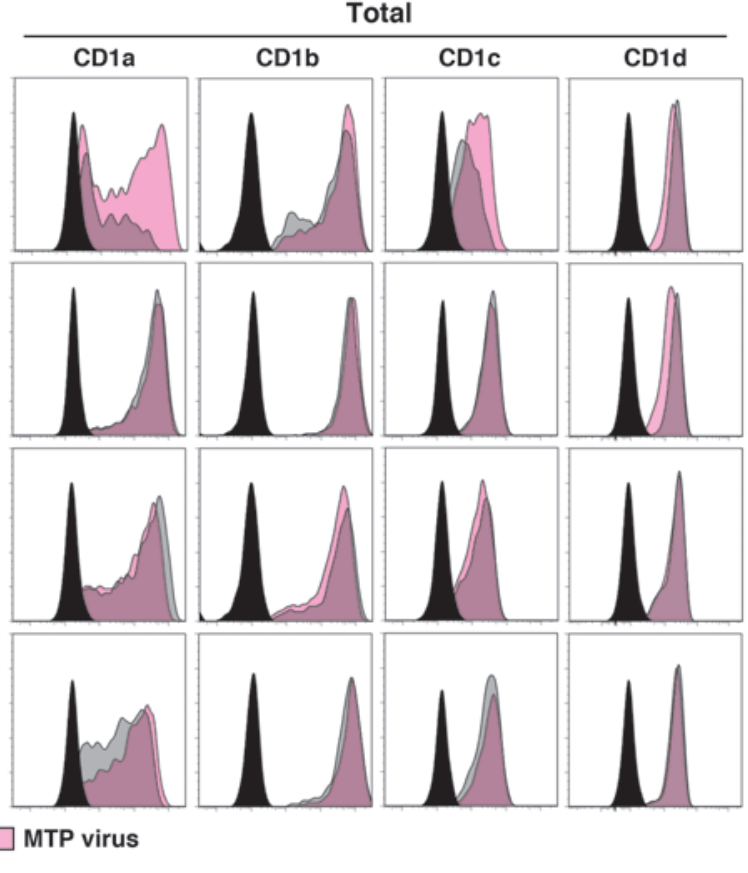

CD1c

B

CD1a
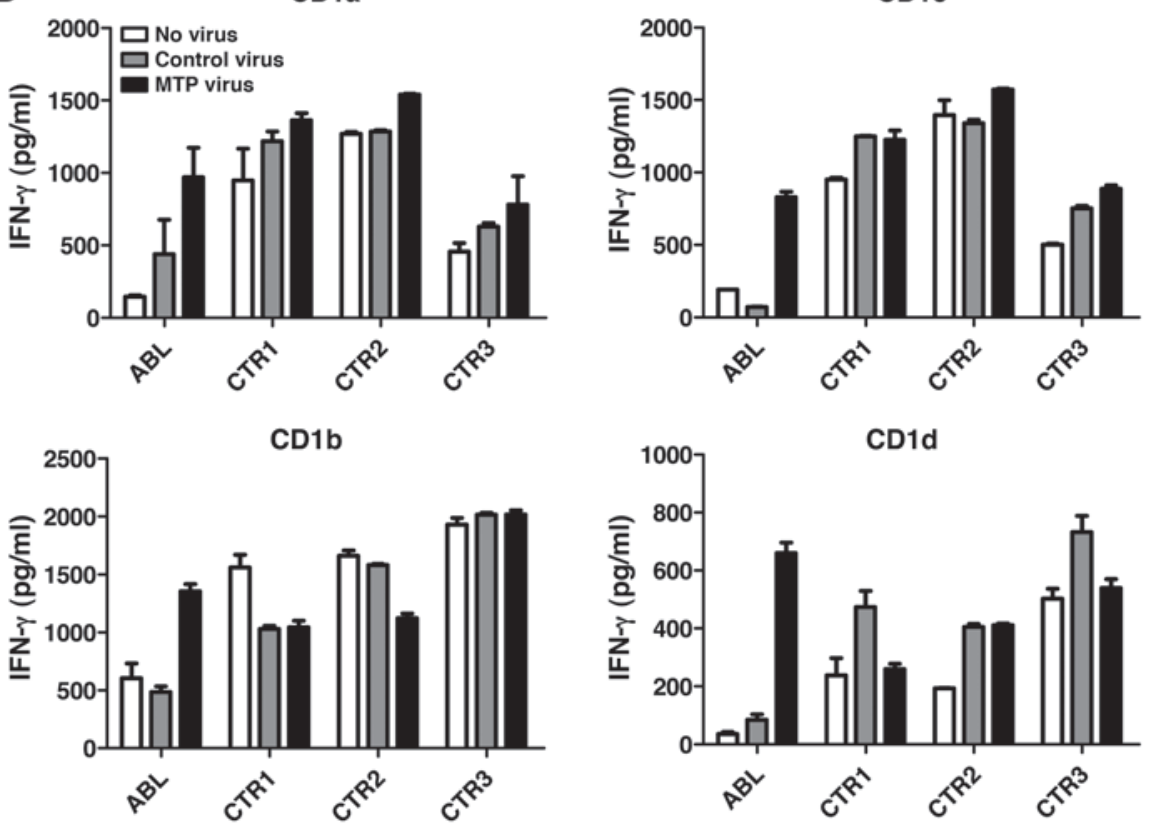

Figure 5

Restoration of CD1 expression and function in ABL upon MTP reconstitution. (A) Flow cytometric analysis of CD1 expression on ABL or control (CTR) DCs 3 days after infection with lentiviruses expressing MTP or the empty control virus. Shown is the mean fluorescence intensity on a log ${ }_{10}$ scale. (B) Analysis of CD1-restricted presentation of self lipids 3 days after infection with lentiviruses expressing MTP or the empty control virus. DCs from healthy controls or ABL patients were cocultured with CD1-restricted T cells or iNKT cells. One representative experiment is shown. One additional experiment with cells from independent $\mathrm{ABL}$ and control subjects showed comparable results.

under steady-state conditions. However, all inhibitors increased group 1 CD1 but not MHC class I or II expression in ABL DCs (Figure 4B and data not shown). In addition, proteasomal inhibition had no effect on CD1d levels, consistent with unaltered CD1d expression in ABL (data not shown). Due to the limited amount of primary DCs obtained from patients and controls, direct determination of the ubiquitin status of CD1 proteins was not possible. However, in contrast to the observations made upon interference with proteasome function, inhibition of lysosomal degradation by concanamycin A- and chloroquine-dependent blockade of acidification did not increase CD1 expression in ABL, although it led to impaired CD1b- and CD1d-restricted presenta- 
A

A $\quad A B L$

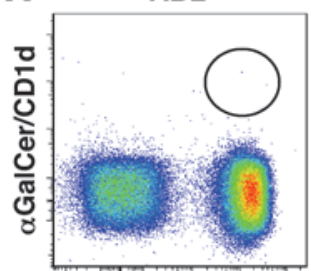

Control 1
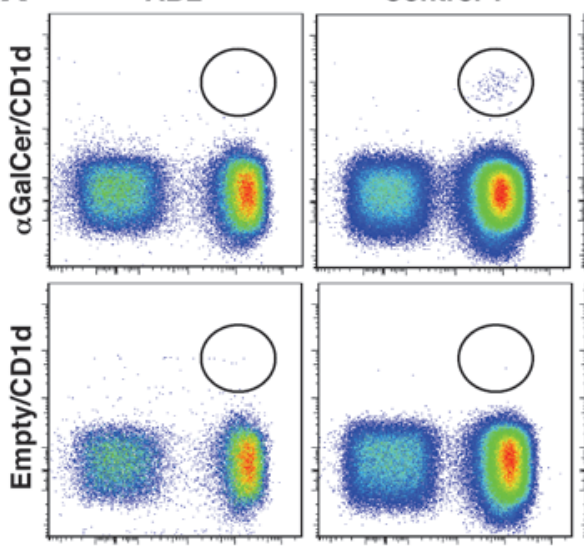

Control 2
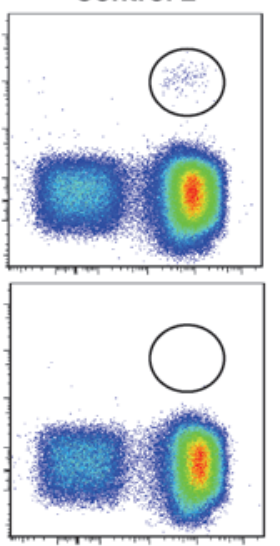

B

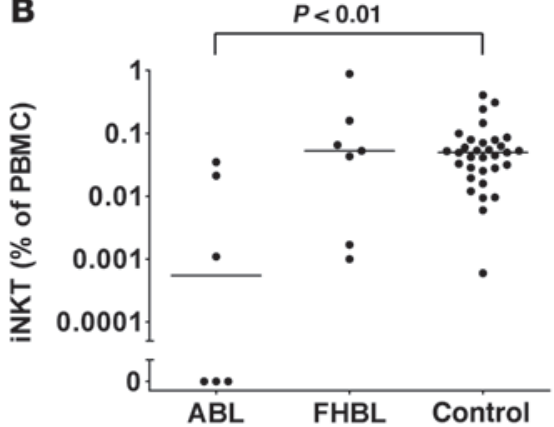

C
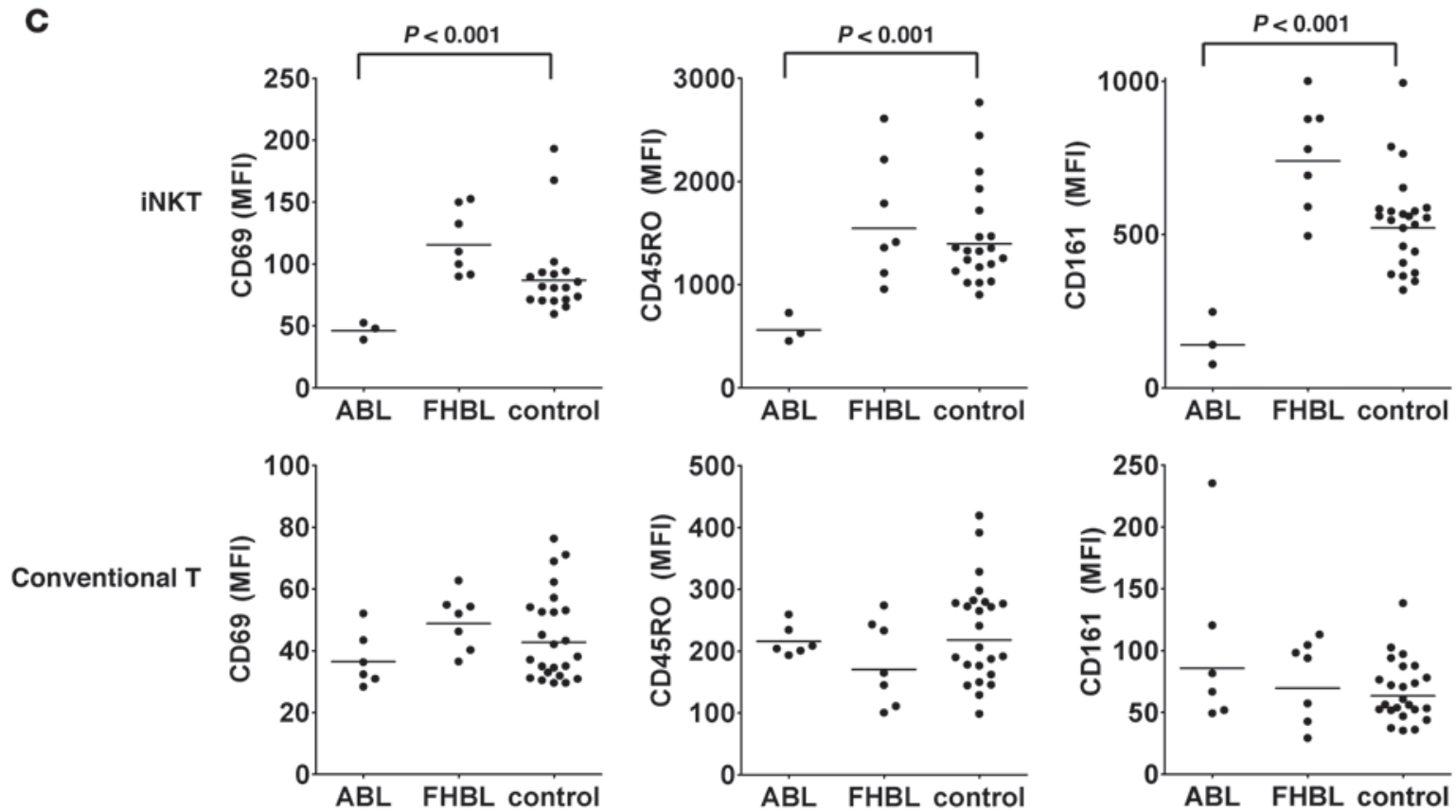

\section{Figure 6}

Reduced iNKT levels and an altered iNKT phenotype in ABL. (A and B) iNKT levels in peripheral blood were determined by staining with $\alpha$ GalCer/CD1d tetramers or empty CD1d tetramers as control and are expressed as percentage of PBMCs. (C) Flow cytometric analysis of expression of cell surface markers on iNKT cells (upper panel) or conventional (tetramer-negative) T cells (lower panel) in peripheral blood. Shown is the mean fluorescence intensity. Dots represent individual subjects. Statistical significance was calculated using the 2-tailed MannWhitney $U$ test. Analysis of iNKT surface markers was limited to patients with detectable iNKT cells. Bars indicate the median.

tion of endolysosome-loaded antigens (data not shown). In conclusion, these data suggest that absence of MTP in the ER leads to proteasomal degradation of group $1 \mathrm{CD} 1$, which is similar to the reported effects of MTP deficiency on APOB $(24,25)$. Interestingly, CD1d shows functional alterations similar to group 1 CD1 but escapes proteasomal degradation.

Reconstitution of $A B L D C$ s with MTP restores $C D 1$ expression and function. To investigate whether reconstitution with wild-type MTP can restore CD1 expression and function, we transduced DCs from healthy controls and ABL patients with lentiviruses expressing MTP. We obtained high transduction rates with unimpaired viability (Supplemental Figure 11). Lentiviral MTP transduction did not affect CD1 expression or antigen presentation in healthy controls
(Figure 5), as previously noted $(6,8)$. In contrast, transduction of ABL DCs largely restored CD1a, CD1b, and CD1c expression, with no effect on MHC class I and II expression (Figure 5A and data not shown). In addition, transduction of ABL DCs with MTP restored CD1-restricted antigen presentation (Figure 5B).

Altered development and peripheral activation of iNKT cells in $A B L$. Since murine thymic iNKT selection requires CD1d (26) and MTP (8), we investigated whether impaired CD1d function in ABL leads to a defect in iNKT selection and reduction in peripheral iNKT numbers. As previously reported (27), we observed considerable variation in peripheral iNKT levels among healthy controls (Figure 6, A and B). However, while all controls and FHBL patients had detectable iNKT cells, levels were significantly reduced in ABL, 
and 3 of 6 patients had undetectable iNKT levels despite analysis of at least $3 \times 10^{6} \mathrm{PBMCs}$. Individual iNKT cell numbers were stable over time (Supplemental Figure 13), confirming that the observed reduction was not caused by time-dependent variation. Interestingly, although all ABL patients showed severe defects in CD1d-restricted antigen presentation (residual CD1d-restricted presentation, $2 \%-17 \%$ of control), lack of peripheral iNKT cells was limited to patients with less than $10 \%$ of residual CD1d function (Supplemental Figure 12). This suggests that a threshold in central CD1d-restricted antigen presentation exists that must be passed to allow for positive selection of iNKT cells. However, due to severe CD1d defects in all ABL patients, subsequent thymic iNKT development was altered even in patients with detectable peripheral iNKT cells, as evidenced by impaired expression of iNKT markers typically acquired during thymic development based upon studies in mouse models (see below) $(28,29)$. These data suggest that MTP deficiency in ABL might lead to thymic CD1d dysfunction and impaired iNKT selection, consistent with similar defects observed in murine thymic organ cultures upon MTP inhibition (30). However, it cannot be excluded that redistribution from blood to other tissues contributes to the reduction in iNKT numbers in ABL.

iNKT cells are effector/memory cells characterized by constitutive expression of activation (CD69), memory (CD45RO), and NK markers (CD161/NK1.1). We analyzed these markers by flow cytometry and found significantly decreased expression of CD69, CD45RO, and CD161 on iNKT cells but not conventional ( $\alpha$ GalCer/CD1d-tetramer-negative) T cells in all ABL patients with detectable iNKT cells (Figure 6C). In addition, the percentage of iNKT cells positive for these markers was decreased in ABL (data not shown). Since CD1d-restricted antigen presentation in the periphery is required for maturation and CD161/NK1.1 acquisition after thymic emigration of NK1.1- iNKT cells (29), these data are consistent with a defect in peripheral CD1d function in ABL in vivo. In addition, maintenance of CD69 expression by iNKT cells is independent of peripheral CD1d (29), and alterations in ABL might therefore reflect impaired thymic iNKT selection, consistent with reduced peripheral iNKT numbers (28). In conclusion, these data demonstrate that impaired CD1-restricted antigen presentation in ABL as observed in vitro is reflected by similar alterations in vivo and is consistent with a defect in both central and peripheral CD1d function.

\section{Discussion}

It has been recognized that metabolism and immunity are highly integrated processes that regulate each other (31). Innate and adaptive immune cells can modulate metabolic pathways, and, conversely, lipid mediators such as prostaglandins, lipoxins, and sphingolipid- and phosphatidylinositol-derived second messengers are crucial regulators of immune function (31). In addition, eukaryotic and prokaryotic lipids can serve as antigens for CD1-restricted lipid antigen presentation, which can be modulated through cellular lipid metabolism $(5,32)$. It has recently been demonstrated that MTP, a protein critically involved in lipoprotein metabolism due to its role in the transfer of lipids onto APOB, is also a central regulator of CD1 function and can directly transfer lipids onto CD1 molecules based upon studies in model systems $(6,7,9)$. Here, we show that MTP deficiency in the rare human disease ABL is characterized by previously unrecognized immune defects, specifically by a loss of lipid antigen presentation by all group 1 and 2 CD 1 mol- ecules. Our studies therefore highlight MTP as a unique regulator of both metabolic and immune functions in humans and suggest that $\mathrm{ABL}$ is not only a primary disorder of lipid metabolism but also an immune disease involving CD1.

Importantly, CD1 dysfunction in $\mathrm{ABL}$ is caused specifically by deficiency in MTP and not by its downstream effects on the metabolism of APOB-containing lipoprotein particles, as demonstrated by unimpaired CD1 function in patients with FHBL caused by heterozygous and compound heterozygous mutations in APOB. In addition, it is noteworthy that expression of apolipoprotein E, an apolipoprotein critical for exogenous CD1-restricted antigen presentation, is unaltered in $\operatorname{ABL}(33,34)$. Furthermore, MTP deficiency in ABL specifically affects CD1 lipid antigen presentation and does not lead to alterations in MHC class I- and class IIrestricted peptide antigen presentation and the activation and phenotype of conventional T cells.

Mechanistically, the lack of functional MTP in ABL is associated with decreased expression of group 1 CD1, likely owing to proteasomal degradation, and leads to a pronounced defect in lipid antigen presentation. These results bear resemblance to classical MHC class I molecules, where interference with loading of self-derived peptides during biosynthesis in the ER causes proteasomal degradation (35). Similarly, loss of MTP function also leads to increased proteasomal degradation of $\operatorname{APOB}(24,25)$. Lack of ER retention of group 1 CD1 molecules in the absence of MTP is likely due to rapid proteasomal degradation preventing intracellular accumulation, similar to prior observations for APOB (36). Our studies therefore demonstrate for the first time to our knowledge that MTP-mediated lipid transfer in the ER is required for unimpaired maturation and survival of group 1 CD1 molecules, which otherwise undergo proteasomal degradation. This is in accordance with the notion that endogenous lipid antigens might stabilize nascent CD1 molecules during biosynthesis (5).

Interestingly, while group 1 CD 1 expression is considerably reduced in $\mathrm{ABL}$ as shown here, the $\mathrm{CD} 1$ molecules that presumably escape proteasomal degradation exhibit a normal intracellular distribution of expression. It is therefore possible that these CD1 molecules are stabilized by endogenous ligands that are loaded in an MTP-independent manner, either spontaneously or facilitated by other, yet-to-be-characterized lipid transfer molecules. This might also explain why some CD1 family members, such as CD1b, appear to be less affected by MTP deficiency than others (Supplemental Figure 1).

$\mathrm{CD} 1 \mathrm{~b}$ maintains its ability to present at least some antigens that are acquired in late endosomal compartments such as GMM C80. These results suggest that the acidic $\mathrm{pH}$ and/or the presence of late endosomal and lysosomal lipid editing molecules might provide a mechanism for survival of a fraction of CD1b molecules. Thus, the defect in group 1 CD1 function appears to mainly affect antigens acquired in the secretory and more superficial compartments within the endolysosomal system.

Intriguingly, CD1d shows unimpaired cell surface expression despite a pronounced functional defect and inability to load exogenous antigens. MHC class II-restricted peptide processing is unaltered in $\mathrm{ABL}$, and the CD1d defect is independent of endolysosomal trafficking. It is therefore unlikely that impaired loading of CD1d is simply the consequence of alterations in lysosomal processing. Alternatively, MTP deficiency during CD1d biosynthesis might lead to structural CD1d changes that prevent subsequent antigen loading. While lack of proteasomal degrada- 
tion and unaltered CD1d expression seem to argue against this possibility, staining of murine MTP-deficient APCs with a panel of more than 10 antibodies indeed revealed lack of CD1d recognition by a selected subset of antibodies, consistent with structural CD1d changes (R.S. Blumberg, unpublished observations). Interestingly, these changes were also not associated with CD1d degradation. In addition, investigations of several lysosomal processing disorders including deficiencies in saposin, cathepsin, and glucocerebrosidase have demonstrated similar results, with unimpaired CD1d expression despite severe functional defects (37-39). Finally, it was recently shown that only a minor subset of endogenous human CD1d-binding ligands are true antigens that lead to iNKT cell activation $(40,41)$. It is therefore possible that CD1d ligands and antigens differ in their requirement for MTP for loading onto CD1d. This could explain unaltered CD1d expression and subcellular distribution despite severe defects in CD1d-restricted antigen presentation, since MTP-independent ligands might stabilize CD1d during biosynthesis, assuring normal maturation and trafficking, but might lack antigenicity and thus the potential to activate NKT cells. In conclusion, the examination of CD1 function in ABL has revealed new insights into the manner in which MTP deficiency affects the expression and function of the various CD1 isoforms.

CD1-restricted lipid antigen presentation is involved in host antimicrobial defense, anticancer immunity, and the regulation of many autoinflammatory diseases $(10,11,18)$. Owing to the lack of known Mendelian disorders of CD1 and NKT deficiency in humans, most of this evidence is derived from the analysis of mouse models and has not been confirmed in humans. In addition, all in vivo studies have been limited to the analysis of CD1d, since mice lack expression of group 1 CD 1 molecules. As a consequence, there is little information available on the biological significance of CD1-restricted lipid antigen presentation in humans (10) Here, we describe the first human disease to our knowledge that is characterized by pronounced defects in all CD1 family members. As such, ABL provides unique insight into the biological role of human CD1. Interestingly, while only a minority of the 100 known ABL cases has been characterized in detail, several potentially immune-related clinical disorders have been described, including cardiac insufficiency caused by lesions resembling chronic interstitial myocarditis $(1,42,43)$, fatal recurrent pulmonary infections (44), and malignancies that are otherwise rarely observed (45-47). Although a causal relationship between these defects in ABL and impaired CD1 function is speculative, these phenomena are consistent with a CD1-associated defect, given the observed role of NKT cells in the defense against several cardiotropic (e.g., Coxsackie viruses) and pulmonary pathogens (e.g., Streptococcus pneumoniae, Pseudomonas aeruginosa, respiratory syncytial virus), as well as in anticancer immunity in mouse models (18). Our studies therefore raise the possibility that the clinical observations described above in $\mathrm{ABL}$ are related to $\mathrm{CD} 1$-associated immune abnormalities, which warrants further investigation.

The selective nature of potentially immune-related defects reported in $\mathrm{ABL}$ is not surprising. First, it is well known that patients deficient in NK cells, TLRs, and TLR adaptors exhibit susceptibility to selected microorganisms with otherwise unaltered immune function (48-50). This is in contrast to broad immunodeficiency reported for inbred mice with similar genetic defects and suggests that compensating mechanisms exist in humans that prevent generalized immunodeficiency (51). Second, CD1 defects in $\mathrm{ABL}$ are not complete in the case of $\mathrm{CD} 1 \mathrm{~b}$, which maintains its ability to present at least some antigens acquired in the deep endolysosomal system. Microorganisms such as M. tuberculosis that express CD1b-restricted antigens loaded in these compartments (17) might therefore elicit only minimally impaired CD1b-restricted immune responses in ABL. Finally, the low prevalence of ABL prevents the analysis of susceptibility to pathogens dependent upon CD1-mediated immune responses but that infrequently lead to infections in humans (e.g., Borrelia burgdorferi or Leishmania spp.) (52-54). Notably, however, increased susceptibility to herpes viruses, a group of common pathogens, has not been reported in $\mathrm{ABL}$. This is surprising given the dramatic defects in CD1d and CD1d-restricted iNKT cells in ABL and previous descriptions of EBV-induced X-linked lymphoproliferative syndrome (XLP) and disseminated Varicella zoster virus $(\mathrm{VZV})$ infection in association with iNKT deficiency (55-58). Since we were unable to examine antiviral immunity in $\mathrm{ABL}$, this discrepancy is unexplained but may suggest that these previously described susceptibilities to herpes virus infections are due to other immune defects either alone or in combination with iNKT deficiency (55-57).

In addition, abnormal CD1 function may not only be directly involved in the immune-related defects described in ABL but may also contribute significantly to the metabolic alterations observed in this disorder. For example, iNKT cells have been shown to drive hyperlipidemia and atherosclerosis but protect from hepatic steatosis $(32,59-62)$. Although the effects of MTP deficiency on lipoprotein metabolism are sufficient to explain both lack of atherosclerosis and frequent hepatic steatosis in ABL (1), it is possible that CD1 and NKT dysfunction may contribute to these phenomena. Our findings therefore indicate that further investigation of the role of iNKT cells in the metabolic processes associated with $\mathrm{ABL}$ is warranted.

In conclusion, our studies show that the function of both of the known substrates for MTP, APOB (24) and CD1 (6), is dramatically disabled in $\mathrm{ABL}$ and highlight the importance of future studies investigating the role of CD1 defects in potentially immune-related phenomena associated with ABL. In addition, since CD1d-restricted NKT cells are implicated in diseases such as atherosclerosis $(59,60)$, inflammatory bowel disease (IBD) (63), and autoimmune hepatitis (64), our studies further raise the possibility that chemical inhibitors of MTP might be suitable not only for the treatment of hyperlipidemic disorders (65) but also for interference with CD1-restricted lipid antigen presentation in human immune-mediated diseases.

\section{Methods}

Study population. The study was approved by the ethics committees of Washington University, University of Pennsylvania, and Brigham and Women's Hospital, and all subjects provided written informed consent. We studied 6 subjects with ABL caused by mutations in the MTTP gene (coding for MTP); 5 FHBL subjects with heterozygous $A P O B$ mutations; 1 homozygous FHBL subject with compound germline mutations in $A P O B$; and 1 severely hypolipidemic FHBL subject with a combination of heterozygous mutations in MTTP, SAR1B, and APOB. Clinical and genetic data are summarized in Supplemental Table 1. Of the 33 healthy normolipidemic controls studied, 16 were male and 17 female (median age, 35; range, 24-56).

Collection and preparation of the samples. Peripheral blood $(80 \mathrm{ml})$ was drawn from nonfasting subjects and shipped overnight at room temperature in BD Vacutainer tubes containing EDTA (BD Biosciences) before extraction of PBMCs as described below. Yield, viability, and function of PBMCs were not affected by storage for up to 48 hours at room temperature (data not shown). 
Cell culture. Cells were maintained in RPMI 1640 supplemented with $10 \%$ FCS, $2 \mathrm{mM}$ L-glutamine, $100 \mathrm{U} / \mathrm{ml}$ penicillin, and $100 \mu \mathrm{g} / \mathrm{ml}$ streptomycin (RPMI-10). Monocytes and B cells were extracted from PBMCs by positive selection using CD14 and CD19 magnetic beads, respectively (Miltenyi Biotec). For generation of monocyte-derived DCs, monocytes were cultured at $1 \times 10^{6}$ cells/ml for 5 days in RPMI-10 medium supplemented with $500 \mathrm{U} /$ $\mathrm{ml}$ recombinant hIL-4 and 1,000 U/ml recombinant hGM-CSF (PeproTech). $\mathrm{T}$ cell clones were maintained in RPMI-10 with $100 \mathrm{U} / \mathrm{ml}$ recombinant hIL-2 (NIH AIDS Research and Reference Reagent Program) with periodic restimulation by irradiated allogeneic feeder PBMCs and phytohemagglutinin (PHA), as described previously (22). SPF3 cells were provided by Rosa Bacchetta (San Raffaele Telethon Institute for Gene Therapy, Milan, Italy), Maria Grazia Roncarolo (San Raffaele Telethon Institute for Gene Therapy), and Craig Morita (University of Iowa, Iowa City, Iowa, USA).

Flow cytometry. For a detailed description of flow cytometry and antibodies, refer to Supplemental Methods. Briefly, cells were preincubated with FcR blocking reagent (Miltenyi Biotec) before staining with monoclonal antibodies for 30 minutes at $4^{\circ} \mathrm{C}$. Staining with CD1d- $\alpha$ GalCer tetramers (NIH Tetramer Core Facility) to detect iNKT was performed for 1 hour at $4^{\circ} \mathrm{C}$. Cells were analyzed using a 4-laser LSRII flow cytometer (BD Biosciences) and FlowJo software (Tree Star Inc.). Because of the low frequency of iNKT cells in peripheral blood, at least $3 \times 10^{6}$ cells were recorded per sample. All experiments were performed with the same lot of antibody under standardized conditions to allow for comparison of mean fluorescence intensities between experiments.

Antigen presentation assays. For a detailed description of antigen presentation assays, refer to Supplemental Methods. Antigen presentation was analyzed by in vitro coculture of CD1-restricted $\mathrm{T}$ cell clones with APCs obtained from peripheral blood of ABL patients, FHBL patients, or healthy controls. CD1d presentation was analyzed using monocytes, monocyte-derived DCs, and B cells, while CD1a-c presentation was analyzed using DCs, the only cell type expressing appreciable levels of group 1 CD1. Samples from each ABL and FHBL patient were processed along with those from 3 healthy controls with which results were compared. APCs were incubated with lipids before washing and coculture with $\mathrm{T}$ cell clones in 96-well flat-bottom plates. In some cases, blocking antibodies were added for 2 hours at $37^{\circ} \mathrm{C}$ before addition of T cells. CD1-restricted lipids and T cells were provided by T.-Y. Cheng (Brigham and Women's Hospital), D.B. Moody (Brigham and Women's Hospital), and M.B. Brenner (Brigham and Women's Hospital). Pattern recognition receptor-dependent, CD1d-restricted antigen presentation was studied by coculture of DCs and J3N.5 iNKT cells in the presence of heat-inactivated S. typhimurium CS015 (66) or S. aureus at an MOI of 20 as described in ref. 22. To study M. tuberculosis-induced lipid antigen presentation, DCs were infected with M. tuberculosis $\mathrm{H} 37 \mathrm{Rv}$ at an MOI of 10 for 2 hours at $37^{\circ} \mathrm{C}$. Extracellular bacteria were removed by low-speed centrifugation $(300 \mathrm{~g})$ of DCs for 5 minutes. DCs were cultured for the indicated times before washing and coculture with $\mathrm{T}$ cells. Cytokine concentrations in the supernatant were determined by ELISA.

Lentiviral MTP reconstitution. Human MTP was amplified using the primers BamHI hMTP_for 5'-GATGGATCCAATATGGGAATTCTTCTTGCT-
GTGCTT-3' and XhoI hMTP_rev 5'-GAACTCGAGTCAAAACCATCCGCTGGAAGT-3' (restriction sites underlined) and $5^{\prime}$ of the IRES-eGFP encoding sequence cloned into the previously described trip/SIN-encoding lentiviral transfer plasmid (67) Lentiviral particles were generated and titered as described in Supplemental Methods. On day 3, DCs were infected with lentiviruses expressing hMTP or the control virus at an MOI of 15 in RPMI-1640 containing $10 \mu \mathrm{g} / \mathrm{ml}$ protamine sulfate. Transduced DCs were cultured at $10^{6}$ cells/ml until day 5 .

Quantitative real-time PCR, immunofluorescence staining, confocal laser scanning microscopy. Immunofluorescence staining, confocal microscopy, RNA extraction, reverse transcription, and quantitative PCR were performed as described in Supplemental Methods. Primer sequences were obtained from PrimerBank (http://pga.mgh.harvard.edu/primerbank/), and sequences are listed in Supplemental Table 2.

Statistics. Data are expressed as mean \pm SEM. For analysis of peripheral iNKT cells and surface marker expression, the median is shown, and statistical significance was determined using the 2-tailed Mann-Whitney $U$ test for comparison of 2 groups. Statistical analyses were done with Prism (GraphPad) software. A $P$ value less than 0.05 was considered significant.

\section{Acknowledgments}

We acknowledge the help and support of the patients and their families that contributed to this study. The authors also acknowledge the help and assistance of Maria Teresa Minaya and the assistance of Gus Schonfeld, Pin Yue Peter Green, and Richard Fedorak with the recruitment and study of FHBL subjects. We thank D. Branch Moody (Brigham and Women's Hospital) and Michael B. Brenner (Brigham and Women's Hospital) for very helpful discussions. This work was supported by NIH grants DK-51362, DK-44319, and DK-53056 and the Harvard Digestive Diseases Center (to R.S. Blumberg); the Deutsche Forschungsgemeinschaft (Ze 814/1-1) and the Crohn's and Colitis Foundation of America (to S. Zeissig); NIH grants HL-38180, DK-56260, the Washington University Digestive Disease Center DK52574 (to N.O. Davidson); and NIH grant DK-066917 (to M.A. Exley). G.S. Besra acknowledges support in the form of a Personal Research Chair from James Badrick, a Royal Society Wolfson Research Merit Award, and the Medical Council and The Wellcome Trust (084923/ $\mathrm{B} / 08 / 7$ ) and is a former Lister Institute-Jenner Research Fellow.

Received for publication February 17, 2010, and accepted in revised form May 10, 2010.

Address correspondence to: Richard S. Blumberg, Division of Gastroenterology, Hepatology, and Endoscopy, Department of Medicine, Brigham and Women's Hospital, Harvard Medical School, 75 Francis Street, Boston, Massachusetts 02115, USA. Phone: 617.732.6917; Fax: 617.264.5185; E-mail: rblumberg@partners.org.

Sebastian Zeissig's present address is: Department of Internal Medicine, University Hospital Schleswig-Holstein, Campus Kiel, Kiel, Germany.

\footnotetext{
1. Berriot-Varoqueaux N, Aggerbeck LP, SamsonBouma M, Wetterau JR. The role of the microsomal triglyceride transfer protein in abetalipoproteinemia. Annu Rev Nutr. 2000;20:663-697.

2. Blasiole DA, Davis RA, Attie AD. The physiological and molecular regulation of lipoprotein assembly and secretion. Mol Biosyst. 2007;3(9):608-619.

3. Raabe M, et al. Analysis of the role of microsomal triglyceride transfer protein in the liver of tissue-specific knockout mice. J Clin Invest. 1999;103(9):1287-1298.
}

4. Raabe M, et al. Knockout of the abetalipoproteinemia gene in mice: reduced lipoprotein secretion in heterozygotes and embryonic lethality in homozygotes. Proc Natl Acad Sci U S A. 1998;95(15):8686-8691.

5. Barral DC, Brenner MB. CD1 antigen presentation: how it works. Nat Rev Immunol. 2007;7(12):929-941.

6. Dougan SK, et al. Microsomal triglyceride transfer protein lipidation and control of CD1d on antigenpresenting cells. J Exp Med. 2005;202(4):529-539.

7. Brozovic S, et al. CD1d function is regulated by microsomal triglyceride transfer protein. Nat Med. 2004;10(5):535-539.

8. Dougan SK, Rava P, Hussain MM, Blumberg RS. MTP regulated by an alternate promoter is essential for NKT cell development. J Exp Med. 2007; 204(3):533-545.

9. Kaser A, et al. Microsomal triglyceride transfer protein regulates endogenous and exogenous antigen presentation by group 1 CD 1 molecules. Eur J Immunol. 2008;38(8):2351-2359. 
10. Bendelac A, Savage PB, Teyton L. The biology of NKT cells. Annu Rev Immunol. 2007;25:297-336.

11. Wu L, Van Kaer L. Natural killer T cells and autoimmune disease. Curr Mol Med. 2009;9(1):4-14.

12. Hava DL, et al. Evasion of peptide, but not lipid antigen presentation, through pathogen-induced dendritic cell maturation. Proc Natl Acad Sci U S A. 2008;105(32):11281-11286.

13. Chen $X$, et al. Distinct endosomal trafficking requirements for presentation of autoantigens and exogenous lipids by human CD1d molecules. J Immunol. 2007;178(10):6181-6190.

14. Prigozy TI, et al. Glycolipid antigen processing for presentation by CD1d molecules. Science. 2001; 291(5504):664-667.

15. Yu KO, et al. Modulation of CD1d-restricted NKT cell responses by using $\mathrm{N}$-acyl variants of alphagalactosylceramides. Proc Natl Acad Sci U S A. 2005; 102(9):3383-3388.

16. McCarthy C, et al. The length of lipids bound to human CD1d molecules modulates the affinity of NKT cell TCR and the threshold of NKT cell activation. J Exp Med. 2007;204(5):1131-1144.

17. Moody DB, et al. Lipid length controls antigen entry into endosomal and nonendosomal pathways for CD1b presentation. Nat Immunol. 2002;3(5):435-442.

18. Tupin E, Kinjo Y, Kronenberg M. The unique role of natural killer $\mathrm{T}$ cells in the response to microorganisms. Nat Rev Microbiol. 2007;5(6):405-417.

19. Kinjo Y, et al. Natural killer T cells recognize diacylglycerol antigens from pathogenic bacteria. Nat Immunol. 2006;7(9):978-986.

20. Kinjo Y, et al. Recognition of bacterial glycosphingolipids by natural killer T cells. Nature. 2005;434(7032):520-525.

21. Moody DB, et al. CD1c-mediated T-cell recognition of isoprenoid glycolipids in Mycobacterium tuberculosis infection. Nature. 2000;404(6780):884-888.

22. Brigl M, Bry L, Kent SC, Gumperz JE, Brenner MB Mechanism of CD1d-restricted natural killer T cell activation during microbial infection. Nat Immunol. 2003;4(12):1230-1237.

23. Mattner J, et al. Exogenous and endogenous glycolipid antigens activate NKT cells during microbial infections. Nature. 2005;434(7032):525-529.

24. Benoist F, Grand-Perret T. Co-translational degradation of apolipoprotein B100 by the proteasome is prevented by microsomal triglyceride transfer protein. Synchronized translation studies on HepG2 cells treated with an inhibitor of microsomal triglyceride transfer protein. J Biol Chem. 1997; 272(33):20435-20442.

25. Brodsky JL, Fisher EA. The many intersecting pathways underlying apolipoprotein B secretion and degradation. Trends Endocrinol Metab. 2008; 19(7):254-259.

26. Gapin L, Matsuda JL, Surh CD, Kronenberg M. NKT cells derive from double-positive thymocytes that are positively selected by CD1d. Nat Immunol. 2001; 2(10):971-978

27. Lee PT, Putnam A, Benlagha K, Teyton L, Gottlieb PA, Bendelac A. Testing the NKT cell hypothesis of human IDDM pathogenesis. J Clin Invest. 2002; 110(6):793-800.

28. Benlagha K, Wei DG, Veiga J, Teyton L, Bendelac A.
Characterization of the early stages of thymic NKT cell development. J Exp Med. 2005;202(4):485-492.

29. McNab FW, et al. The influence of CD1d in postselection NKT cell maturation and homeostasis. J Immunol. 2005;175(6):3762-3768.

30. Dougan SK, Kaser A, Blumberg RS. CD1 expression on antigen-presenting cells. Curr Top Microbiol Immunol. 2007;314:113-141.

31. Hotamisligil GS. Inflammation and metabolic disorders. Nature. 2006;444(7121):860-867.

32. Yang L, Jhaveri R, Huang J, Qi Y, Diehl AM. Endoplasmic reticulum stress, hepatocyte CD1d and NKT cell abnormalities in murine fatty livers. $L a b$ Invest. 2007;87(9):927-937.

33. Blum CB, Deckelbaum RJ, Witte LD, Tall AR, Cornicelli J. Role of apolipoprotein E-containing lipoproteins in abetalipoproteinemia. J Clin Invest. 1982;70(6):1157-1169.

34. van den Elzen P, et al. Apolipoprotein-mediated pathways of lipid antigen presentation. Nature. 2005;437(7060):906-910.

35. Hughes EA, Hammond C, Cresswell P. Misfolded major histocompatibility complex class I heavy chains are translocated into the cytoplasm and degraded by the proteasome. Proc Natl Acad SciUS A. 1997;94(5):1896-1901.

36. Liao W, Hui TY, Young SG, Davis RA. Blocking microsomal triglyceride transfer protein interferes with apoB secretion without causing retention or stress in the ER. J Lipid Res. 2003;44(5):978-985

37. Balreira A, Lacerda L, Miranda CS, Arosa FA. Evidence for a link between sphingolipid metabolism and expression of CD1d and MHC-class II: monocytes from Gaucher disease patients as a model. BrJ Haematol. 2005;129(5):667-676.

38. Honey K, et al. Thymocyte expression of cathepsin $\mathrm{L}$ is essential for NKT cell development. Nat Immunol. 2002;3(11):1069-1074.

39. Yuan W, et al. Saposin B is the dominant saposin that facilitates lipid binding to human CD1d molecules. Proc Natl Acad Sci U S A. 2007;104(13):5551-5556

40. Fox LM, et al. Recognition of lyso-phospholipids by human natural killer T lymphocytes. PLoS Biol. 2009; 7(10):e1000228.

41. Cox D, et al. Determination of cellular lipids bound to human CD1d molecules. PLoS One. 2009;4(5):e5325.

42. Dische MR, Porro RS. The cardiac lesions in Bassen-Kornzweig syndrome. Report of a case, with autopsy findings. Am J Med. 1970;49(4):568-571.

43. Sobrevilla LA, Goodman ML, Kane CA. Demyelinating central nervous system disease, macular atrophy and acanthocytosis (Bassen-Kornzweig Syndrome). Am JMed. 1964;37:821-828.

44. Becroft DM, Costello JM, Scott PJ. A-beta-lipoproteinaemia (Bassen-Kornzweig syndrome): report of a case. Arch Dis Child. 1965;40:40-46.

45. Al-Shali K, Wang J, Rosen F, Hegele RA. Ileal adenocarcinoma in a mild phenotype of abetalipoproteinemia. Clin Genet. 2003;63(2):135-138.

46. Bieri JG, Hoeg JM, Schaefer EJ, Zech LA, Brewer HB Jr. Vitamin A and vitamin E replacement in abetalipoproteinemia. Ann Intern Med. 1984;100(2):238-239.

47. Newman RP, Schaefer EJ, Thomas CB, Oldfield EH. Abetalipoproteinemia and metastatic spinal cord glioblastoma. Arch Neurol. 1984;41(5):554-556.
48. Orange JS. Human natural killer cell deficiencies and susceptibility to infection. Microbes Infect. 2002; 4(15):1545-1558

49. von Bernuth $\mathrm{H}$, et al. Pyogenic bacterial infections in humans with MyD88 deficiency. Science. 2008; 321(5889):691-696.

50. Zhang SY, et al. TLR3 deficiency in patients with herpes simplex encephalitis. Science. 2007; 317(5844):1522-1527.

51. Casanova JL, Abel L. Primary immunodeficiencies: a field in its infancy. Science. 2007;317(5838):617-619.

52. Tupin E, et al. NKT cells prevent chronic joint inflammation after infection with Borrelia burgdorferi. Proc Natl Acad Sci US A. 2008;105(50):19863-19868.

53. Amprey JL, et al. A subset of liver NK T cells is activated during Leishmania donovani infection by CD1d-bound lipophosphoglycan. J Exp Med. 2004; 200(7):895-904.

54. Mattner J, Donhauser N, Werner-Felmayer G, Bogdan C. NKT cells mediate organ-specific resistance against Leishmania major infection. Microbes Infect. 2006;8(2):354-362.

55. Nichols KE, et al. Regulation of NKT cell development by SAP, the protein defective in XLP. Nat Med. 2005;11(3):340-345.

56. Rigaud S, et al. XIAP deficiency in humans causes an X-linked lymphoproliferative syndrome. Nature. 2006;444(7115):110-114

57. Pasquier B, et al. Defective NKT cell development in mice and humans lacking the adapter SAP, the $\mathrm{X}$-linked lymphoproliferative syndrome gene product. J Exp Med. 2005;201(5):695-701.

58. Levy $\mathrm{O}$, et al. Disseminated varicella infection due to the vaccine strain of varicella-zoster virus, in a patient with a novel deficiency in natural killer $\mathrm{T}$ cells. J Infect Dis. 2003;188(7):948-953.

59. Tupin E, et al. CD1d-dependent activation of NKT cells aggravates atherosclerosis. J Exp Med. 2004; 199(3):417-422.

60. Nakai Y, et al. Natural killer T cells accelerate atherogenesis in mice. Blood. 2004;104(7):2051-2059.

61. Elinav E, et al. Adoptive transfer of regulatory NKT lymphocytes ameliorates non-alcoholic steatohepatitis and glucose intolerance in ob/ob mice and is associated with intrahepatic CD8 trapping. J Pathol. 2006;209(1):121-128.

62. Lo JC, et al. Lymphotoxin beta receptor-dependent control of lipid homeostasis. Science. 2007; 316(5822):285-288

63. Fuss IJ, et al. Nonclassical CD1d-restricted NK T cells that produce IL-13 characterize an atypical Th2 response in ulcerative colitis. J Clin Invest. 2004; 113(10):1490-1497.

64. Swain MG. Hepatic NKT cells: friend or foe? Clin Sci (Lond). 2008;114(7):457-466.

65. Cuchel M, et al. Inhibition of microsomal triglyceride transfer protein in familial hypercholesterolemia. N Engl J Med. 2007;356(2):148-156.

66. Miller SI, Kukral AM, Mekalanos JJ. A two-component regulatory system (phoP phoQ) controls Salmonella typhimurium virulence. Proc Natl Acad Sci US A. 1989;86(13):5054-5058.

67. Breckpot K, et al. Lentivirally transduced dendritic cells as a tool for cancer immunotherapy. J Gene Med. 2003;5(8):654-667. 